\title{
CONSUMO DE DROGAS LÍCITAS E ILÍCITAS EN ESCOLARES Y FACTORES DE PROTECCIÓN Y RIESGO
}

\author{
Carmen Gloria Fraile Duvicq \\ Náyade Riquelme Pereira ${ }^{3}$ \\ Ana Maria Pimenta Carvalho 4
}

Duvicq CGF, Pereira NR, Carvalho AMP. Consumo de drogas lícitas e ilícitas en escolares y factores de protección y riesgo. Rev Latino-am Enfermagem 2004 março-abril; 12(número especial):345-51.

Los objetivos de la investigación fueron identificar la población consumidora de drogas lícitas e ilícitas entre escolares de sexto año básico de escuelas municipalizadas urbanas de Chiguayante, conocer los niveles de riesgo e identificar los factores de riesgo y protección. Estudio descriptivo, transversal, correlacional. El instrumento aplicado a 301 escolares, fue el Dusy Abreviado. Las variables fueron sometidas a análisis estadístico descriptivo - comparativo mediante el test de Chi Cuadrado de Pearson y ANOVA. Hubo 60\% de consumidores de drogas lícitas, iniciados entre los 8 y 11 años. La prevalencia de vida del consumo de tabaco y alcohol fue de 18,7\% y 16,3\% respectivamente. El 85\% de los varones mostró inclinación al consumo, de los cuales 69\% tiene entre 11 y 12 años. Existen preferentemente consumidores de drogas lícitas. Las conductas asociadas al factor de riesgo personal fueron las más relevantes, las de protección se asociaron principalmente al factor protector microsocial. Todos estaban sometidos a diversos niveles de riesgo.

DESCRIPTORES: consumo; drogas lícitas e ilícitas; escolares; factores de riesgo y protección; enfermería

\section{CONSUMPTION OF LICIT AND ILLICIT DRUGS IN STUDENTS AND THE FACTORS OF PROTECTION AND RISK}

The aims of the investigation were identify the population that consumes licit and illicit drugs among students of sixth primary, from municipal urban schools of Chiguayante, know the levels of risks and identify risks factors and protection. Descriptive, transversal, correlate study. The instrument applied to 301 students was the Dusy Abreviado. The variables were subjected to a stadistic descriptive - comparative analysis through the test of Chi - Cuadrado de Pearson and ANOVA.There was 60\% of the consumers of licit drugs, that began consumption between 8 and 11 years. The prevalence of use of tobacco and alcohol was $18,7 \%$ and $16,3 \%$ respectively. The $85 \%$ of men showed inclination to the consumption, from which $69 \%$ is between 11 and 12 years old. There are mainly abusers of licit drugs. The behaviours associated to the personal risk factor were the most relevant, the ones of protection were mainly associated with the micro-social protective factor. All subjects were submitted to different levels of risk.

DESCRIPTORS: consumption; licit and illicit drugs; students; risk and protective factors; nursing

\section{O CONSUMO DE DROGAS LÍCITAS E ILÍCITAS POR ESTUDANTES E FATORES DE PROTEÇÃO E DE RISCO}

O objetivo do presente trabalho foi identificar características dos consomumidores de drogas lícitas e ilícitas entre estudantes de sexto série, das escolas urbanas municipais de Chiguayante, relacionando-as a fatores de risco e de proteção. É um estudo descritivo e correlacional. Utilizou-se, para a coleta dos dados o Dusy Abreviado, com 301 crianças. As variáveis foram sujeitadas à análise comparativa através do teste do chi - quadrado de Pearson e ANOVA. Encontrou-se que 60\% dos consumidores de drogas licitas iniciam o consumo entre 8 e 11 anos. As substâncias mais consumidas foram o álcool e 0 tabaco, $18.7 \%$ e $16.3 \%$ respectivamente. $85 \%$ dos consumidores eram do sexo masculino e, destes, $69 \%$ iniciavam na faixa etária de 11 a 12 anos. Há principalmente abusadores de drogas licitas. Os comportamentos associados ao fator de risco pessoal foram os mais relevantes, e os fatores de proteção mais relevantes encontravam-se na esfera micro-social.

DESCRITORES: drogas lícitas e ilícitas; estudantes; consumo; fatores de risco e de proteção, enfermagem

\footnotetext{
${ }^{1}$ Las opiniones expresadas en este articulo son de responsabilidad exclusiva de los autores y no representan la posición de la organización donde trabajan o de su administración; ${ }^{2}$ Magíster en Bioética, Facultad de Medicina, Universidad de Concepción, correo electrónico: cfraile@udec.cl; ${ }^{3}$ Magíster en Salud Familiar, Facultad de Medicina, Universidad de Concepción, correo electrónico: nriquelm@udec.cl; ${ }^{4}$ Docente de la Escuela de Enfermería de Ribeirão Preto, de la Universidad de São Paulo, Centro Colaborador de la OMS para el desarrollo de la investigación en enfermería, correo electrónico: anacar@eerp.usp.br
} 


\section{INTRODUCCIÓN}

La adolescencia es el comienzo de un período de gran vulnerabilidad por los aspectos biopsicosociales que debe enfrentar el adolescente. Todos comparten el mismo proceso de crecimiento y desarrollo, pero las características sociales y culturales de cada uno influyen notablemente en el proceso de hacerse mayor ${ }^{(1)}$. El proceso de desarrollo social implica para el adolescente cumplir ciertas tareas, entre ellas está establecer una identidad personal y grupal además de separarse gradualmente de los padres ${ }^{(2)}$. Entre los problemas externos que deberá enfrentar el adolescente, se cuentan las drogas. El fenómeno de las drogas es altamente complejo, multicausal, que no reconoce límites territoriales ni sociales, tampoco de edad.

El término droga se utiliza, actualmente, como sinónimo de sustancia psicoactiva que tiene un efecto dañino para el sujeto que la consume $\mathrm{e}^{(3)}$. Por consumo de sustancias se entiende el introducir un producto químico que afecta al organismo, incluidas sustancias legales, ilegales o medicinales $y$, por abuso de sustancias, cuando éstas alteran la percepción del individuo, o sea alteran su sistema nervioso central, pudiendo intensificar o deprimir el estado de ánimo o las emociones ${ }^{(1)}$. La drogodependencia caracterizada por la presencia de signos y síntomas cognitivos, conductuales y fisiológicos indican que el individuo ha perdido el control sobre el uso de sustancias psicoactivas y las sigue consumiendo a pesar de sus consecuencias adversas ${ }^{(3)}$. Sin embargo, se puede precisar que en la aparición del consumo de drogas se combinan tres aspectos, el farmacológico que representa la droga y su poder adictivo, la interacción social, dada por la familia, escuela, comunidad y sociedad y la presencia de un sujeto vulnerable ${ }^{(4)}$.

Un estudio realizado en Chile menciona que el abuso de sustancias ha sido identificado como un problema mayor en la salud mental, física y económica ${ }^{(5)}$. La realidad chilena presenta ciertos problemas de salud mental, como la violencia delictual en el medio escolar y el consumo inmoderado de alcohol, problemas que están ocurriendo cada vez a edades más tempranas ${ }^{(6)}$. En 1995 uno de cada cinco detenidos por delitos era menor de edad, en 1997 era uno de cada tres. Todas las sustancias psicoactivas usadas en forma abusiva producen aumento del riesgo de accidentes y de violencia, particularmente en adolescentes por la baja percepción del riesgo.

Un estudio presentado por el Consejo Nacional para el Control de Estupefacientes (CONACE), presenta conclusiones comparativas de tres períodos 1995, 1997 y 1999, entre ellas una disminución en la edad de inicio del consumo de drogas ilícitas y cierta estabilización en la edad de inicio de drogas lícitas ${ }^{(7)}$. Otro estudio realizado por este mismo organismo presenta un aumento en el consumo de drogas lícitas en el grupo de los 12 a 18 años de edad, particularmente en el consumo de alcohol, el que incrementa de un $41,1 \%$ en 1996 a un $46,8 \%$ en el 2000 , y respecto del consumo de drogas ilícitas, éste aumentó de un $0,6 \%$ a un $5,2 \%$ en igual período ${ }^{(8)}$.

Los adolescentes que presentan posibilidades de abuso de sustancias son los que están sometidos a diferentes factores de riesgos ${ }^{(1)}$. La literatura científica reconoce factores de riesgo psicosociales que afectan a los adolescentes entre los que destacan el ambiente familiar, la influencia de los pares, las destrezas sociales y las características de personalidad ${ }^{(5)}$. Ellos determinan o aumentan la posibilidad del consumo de drogas, pero existen otros que reducen la posibilidad del consumo, conocidos como factores protectores, situados en los ámbitos mencionados. La manera de cómo estos factores operen, no es constante ni estable, su interacción es dinámica y se modifica de manera interactiva durante el curso de la vida ${ }^{(9)}$. Existen factores inductores como problemas de conducta o alteraciones mentales, y factores condicionantes como la curiosidad, la presión del grupo y la disponibilidad de drogas, entre otras, proporcionando condiciones para el consumo.

El uso y abuso de drogas en adolescente genera problemas físicos, agudos o crónicos, a corto, mediano o largo plazo, problemas económicos a la familia y sociedad, también problemas morales asociados por ejemplo a la permisividad sexual. Los problemas en el proceso de aprendizaje se verifican al disminuir la capacidad de concentración, de síntesis y organización del material, de aprender conceptos nuevos, de aplicar principios nuevos a problemas específicos, de enjuiciar tareas y situaciones complejas, $y$ tomar decisiones adecuadas y a tiempo ${ }^{(1)}$. A todo lo anterior incorpora la inhibición del interés por el otro sexo, la apatía social y desinterés por los deportes y otras entretenciones. Por último, "el consumo de drogas impide al adolescente el normal paso de la adolescencia a la edad adulta, pues la droga lo induce a desarrollar una actitud egocéntrica centrada en el presente" ${ }^{\text {(10) }}$

Conciente que la búsqueda de soluciones es de carácter colectivo, este estudio pretende contribuir a la detección precoz de factores de riesgo y de protección, para según corresponda, realizar intervenciones de carácter primario y/o secundario. Para lograr lo anterior 
esta investigación se propuso los siguientes objetivos: identificar la población consumidora de drogas lícitas y ilícitas entre los escolares de sexto año básico de las escuelas municipalizadas de la Comuna de Chiguayante, describir la población escolar y particularmente aquella consumidora según ciertas variables sociodemográficas, identificar factores de riesgo y protección personales y microsociales presentes en los escolares, y finalmente conocer los niveles de riesgo e índices de severidad en torno a las conductas presentadas por los escolares.

\section{MÉTODO}

La investigación realizada fue de tipo descriptivo, transversal y correlacional. El universo estuvo constituido por la totalidad de los escolares de la Comuna de Chiguayante. La población diana estuvo formada por los alumnos de sexto año básico de las escuelas municipalizadas de la Comuna, y la población disponible debía contar con la autorización por escrito de los padres o apoderados además de no pertenecer a hogares infantiles. De esta población accesible, se alcanzó finalmente la muestra cuyo número fue de 301 escolares. El tamaño muestral se obtuvo fijando un $95 \%$ de confianza, y se realizó por medio de una distribución porcentual proporcional a las escuelas, la que consideró remplazar a aquellos alumnos que no desearon participar.

Previo a la investigación se realizaron contactos con la Dirección Comunal de Educación para obtener autorización y antecedentes, con los directores y profesores jefes de cada establecimiento para presentarles el proyecto y solicitarles colaboración. Los profesores jefes de cada curso hicieron llegar una nota informativa a los padres de los alumnos seleccionados, que explicaba brevemente el objetivo de la investigación y la invitación a participar de ella, acompañada del Consentimiento Informado, el que debía retornar firmado. La encuesta fue autoaplicada previa instrucción del docente en una sala exclusiva y dedicando el tiempo requerido por cada adolescente.

La recolección de los datos se obtuvo a través del Cuestionario DUSI Abreviado (Abreviación del Drug Use Screening Inventory) ${ }^{(11)}$. Instrumento diseñado para cuantificar y categorizar los indicadores del consumo de drogas, en relación con el estado físico, emocional, psicosocial, familiar y académico del adolescente. El cuestionario consta de tres partes: primero la identificación del escolar, luego un cuerpo de 48 preguntas que hacen referencia a conductas o acciones problemáticas, las que se agrupan en áreas problemas o escalas, ellas son escala de conducta, emocional, familia, académica, grupo de pares, recreación, agresividad, depresión y drogas. Cuando las interrogantes se presentan siempre o casi siempre o muchas veces, son catalogadas de riesgo. Por el contrario, cuando las respuestas se presentan en las opciones nunca o casi nunca, no representan mayor riesgo. Es posible determinar el índice de severidad de cada escala, lo que equivale al grado de riesgo frente al consumo de drogas. Finalmente una nómina de drogas lícitas e ilícitas, edad y frecuencia del consumo. Entre las ventajas de este cuestionario está su rápida aplicación, y no requerir de entrenamiento previo.

El instrumento fue validado por medio de una prueba piloto practicada a un $10 \%$ del valor de la muestra, a una población distinta pero, con iguales características en edad y tipo de escuela.

Para medir la confiabilidad del instrumento en su totalidad y de cada una de las escalas, se aplicó el coeficiente Alpha de Crombach, el que resultó en su escala global altamente confiable, 0,91 lo que se tradujo en alta consistencia y homogeneidad. En el procesamiento de los datos se utilizó el programa estadístico SPSS en su versión 8.0, el que permitió realizar el análisis estadístico descriptivo y las comparaciones (test de Chi - Cuadrado de Pearson y ANOVA) de las variables en estudio. Estas fueron agrupadas en variables dependientes, consumo de drogas lícitas e ilícitas y las independientes como las sociodemográficas, las asociadas al consumo de drogas lícitas e ilícitas, y las asociadas a conductas de riesgo y de protección.

\section{RESULTADOS}

La distribución por sexo de la muestra fue homogénea, con el $93 \%$ de las edades entre los 11 y 13 años. El último promedio de notas de estos escolares estuvo por sobre el mínimo aceptable, que corresponde a un promedio 4. De los 301 escolares, 293 respondieron la encuesta, de ellos 80 han consumido alguna vez en su vida drogas lícitas, vale decir alcohol y/o tabaco. De los 290 alumnos que respondieron respecto al consumo de drogas ilícitas, (marihuana, cocaína, estimulantes, tranquilizantes, pasta base, heroína e inhalantes) 9 declararon haberlas consumido, y de éstos, 6 presentaron alguna modalidad de consumo de drogas lícitas, significa por lo tanto, que 3 alumnos consumieron sólo alguna vez 
en su vida drogas ilícitas. La prevalencia de vida para estas drogas, fue de $27,3 \%$ y $3,1 \%$ respectivamente.

Según lo anterior, la variable consumo de drogas lícitas fue superior en prevalencia, por ello que el análisis estadístico de las variables sociodemográficas se realizó en torno a ellas. De los 80 alumnos consumidores, 25 sólo consumían alcohol, 32 sólo tabaco y 23 ambas drogas, en tanto que 48 han consumido alcohol y 55 tabaco.

Al combinar las variables consumo de drogas lícitas y la edad de inicio, se verifica que para ambas drogas el consumo fue mayoritariamente entre los 8 y 11 años. La edad de inicio del consumo de ambas drogas lícitas, se presentó según la información obtenida, a los 5 años, dato que resulta poco creíble, pero probable. Situación similar presentan dos escolares que denuncian consumo de ambas drogas lícitas y casi la totalidad de drogas ilícitas, sin especificar la frecuencia ni las edades en que estas últimas fueron consumidas, dato que resulta igualmente poco creíble, pero probable. El $80 \%$ de quienes consumieron alcohol y $60 \%$ de quienes consumieron de tabaco lo hicieron entre estas edades (Tablas 1 y 2 ).

Tabla 1 - Edad que los escolares de sexto año de las escuelas municipalizadas de la comuna de Chiguayante, denuncian haber consumido por primer vez alcohol

\begin{tabular}{lcccccccc}
\hline Consumo Drogas & \multicolumn{9}{c}{ Edad Inicio Consumo Alcohol } \\
Lícitas & \multicolumn{5}{c}{$5-7$} & \multicolumn{1}{c}{$8-11$} & $12-14$ & \multicolumn{2}{c}{ total } \\
& № & $\%$ & № & $\%$ & № & $\%$ & № & $\%$ \\
\hline Alcohol o Tabaco & - & - & 15 & 88,2 & 2 & 11,7 & 17 & 100,0 \\
Alcohol y Tabaco & 3 & 14,4 & 15 & 71,4 & 3 & 14,2 & 21 & 100,0 \\
\hline Total & 3 & 7,9 & 30 & 79,0 & 5 & 13,1 & 38 & 100,0 \\
\hline
\end{tabular}

Fuente: "Consumo de Drogas Lícitas e llícitas en Escolares y Conductas de Protección y Riesgo" Carmen Gloria Freile 2003 Chi Cuadrado de Pearson $=8,828 \quad$ Valor $p=0,357$
Tabla 2 - Edad que los escolares de sexto año de las escuelas municipalizadas de la comuna de Chiguayante, denuncian haber consumido por primera vez tabaco

\begin{tabular}{lcccccccc}
\hline Consumo Drogas & \multicolumn{8}{c}{ Edad Inicio Consumo Tabaco } \\
Lícitas & \multicolumn{2}{c}{$5-7$} & \multicolumn{2}{c}{$8-11$} & $12-13$ & \multicolumn{2}{c}{ total } \\
& № & $\%$ & № & $\%$ & № & $\%$ & № & $\%$ \\
\hline Alcohol o Tabaco & 6 & 22,2 & 13 & 48,1 & 8 & 30,0 & 27 & 100,0 \\
Alcohol y Tabaco & - & - & 15 & 79.0 & 4 & 21,0 & 19 & 100,0 \\
\hline Total & 6 & 13,0 & 28 & 61,0 & 12 & 26,0 & 46 & 100,0 \\
\hline
\end{tabular}

Fuente: Idem Tabla 1 Chi Cuadrado de Pearson $=11,388$ Valor $p=$ 0,123

Existe una alta significación estadística $(p=0,000)$ entre consumo de drogas lícitas y disponibilidad de drogas (referido a accesibilidad), destacando que entre los 80 alumnos consumidores de drogas lícitas, el 30,4\% estaría dispuesto a consumir cualquier droga si estuviera a su alcance, respuesta emitida por aquellos que consumen ambas drogas, y los que consumen sólo una droga lícita $(5,3 \%)$, harían lo mismo al disponer de ellas. Otro hecho destacable es un grupo importante de alumnos vulnerables $(12,6 \%)$, en cuanto posibles consumidores, pues no mostraron una negación al consumo si dispusieran de ellas. Existe también una importante significación estadística entre las variables disponibilidad de drogas y sexo $(p=$ $0,005)$, los varones muestran mayor inclinación hacia el consumo, un $84,6 \%$ a diferencia de las mujeres con un $15,4 \%$. Igualmente existe una alta significación estadística entre las variables disponibilidad de drogas y edad, el $69 \%$ de los que dicen que consumirían si dispusieran de ellas tienen 11 y 12 años (Tabla 3).

Tabla 3 - Edades de los escolares de sexto año básico que consumirían drogas según disponibilidad de ellas en escuelas municipalizadas de la comuna de Chiguayante

\begin{tabular}{|c|c|c|c|c|c|c|c|c|c|c|c|c|c|c|c|c|}
\hline \multirow{3}{*}{$\begin{array}{l}\text { Disponibilidad } \\
\text { De Drogas }\end{array}$} & \multicolumn{16}{|c|}{ Edad de los Escolares de Sexto Año Básico } \\
\hline & \multicolumn{2}{|c|}{ No Regist } & \multicolumn{2}{|c|}{11} & \multicolumn{2}{|c|}{12} & \multicolumn{2}{|c|}{13} & \multicolumn{2}{|c|}{14} & \multicolumn{2}{|c|}{15} & \multirow{2}{*}{$\begin{array}{l}16 \\
\mathrm{~N} \cong\end{array}$} & \multirow[b]{2}{*}{$\%$} & \multicolumn{2}{|l|}{ Total } \\
\hline & $\mathrm{N} \stackrel{0}{-}$ & $\%$ & $\mathrm{~N} \cong$ & $\%$ & $\mathrm{~N} \cong$ & $\%$ & $\mathrm{~N} \stackrel{\circ}{-}$ & $\%$ & $\mathrm{~N} \stackrel{-}{-}$ & $\%$ & $\mathrm{~N} \stackrel{0}{-}$ & $\%$ & & & & $\%$ \\
\hline Sí consumiría & - & - & 2 & 15,4 & 7 & 53,8 & - & - & 1 & 7,7 & 3 & 23,1 & - & - & 13 & 100 \\
\hline No consumiría & 4 & 1,6 & 117 & 47,0 & 93 & 37,3 & 24 & 9,6 & 10 & 4,0 & - & - & 1 & 0,4 & 249 & 100 \\
\hline No responden & - & - & 15 & 38,5 & 20 & 51,3 & 2 & 5,1 & 2 & 5,1 & - & - & - & - & 39 & 100 \\
\hline Total & 4 & 1,3 & 134 & 44,5 & 120 & 39,9 & 26 & 8,6 & 13 & 4,3 & 3 & 1,0 & 1 & 0,3 & 301 & 100 \\
\hline
\end{tabular}

Fuente: Idem Tabla $1 \quad$ Chi Cuadrado de Pearson $=75,377$

Valor $p=0,000$

La frecuencia del consumo de alcohol mensual, fue posible calcularlo al $50 \%$ de los alumnos consumidores, en tanto que a los de tabaco sólo a un $45 \%$, ya que el resto no respondió la interrogante. La alternativa más reiterada al medir la frecuencia del consumo de alcohol fue, sólo una vez al mes, con un $33,8 \%$ y en el caso del tabaco la misma opción se presentó en un 30,8\%, comparada con la alternativa, más de una vez al mes, que para el alcohol fue de 16,3\% mientras que para el tabaco un $25,1 \%$ ( $p=0,000$ para la frecuencia consumo alcohol último mes y $p=0,064$ frecuencia consumo tabaco último mes).

Se identificaron conductas de riesgo, entendidas como acciones o actitudes que pueden facilitar mayor inclinación al consumo de drogas lícitas o ilícitas y poner la salud del adolescente en dificultades a corto, mediano 
o largo plazo, a nivel personal y micro social, a partir de los porcentajes más elevados encontrados en los escolares consumidores y no consumidores. Así, entre aquellos que han consumido drogas lícitas las conductas de riesgo más frecuentes fueron aquellas que hacían referencia a factores de riesgo personales, a diferencia de aquellos no consumidores, cuyas conductas de exentas riesgo, traducidas en conductas protectoras, (acciones o actitudes en orden a potenciar o mantener la salud del adolescente, que pueden reducir la inclinación al consumo de drogas) hacían referencia a factores protectores micro sociales (Tablas 4 y 5 ).

Tabla 4-Conductas de riesgo personales más frecuentes entre los escolares consumidores de drogas lícitas de sexto año básico de las escuelas municipalizadas de la comuna de Chiguayante

\begin{tabular}{lccccc}
\hline \multirow{2}{*}{ Conductas } & \multicolumn{5}{c}{ Consumidores de Drogas } \\
& Sin Riesgo & \multicolumn{2}{c}{ Riesgo } & Recuento \\
& $\mathrm{R}$ & $\%$ & $\mathrm{R}$ & $\%$ & $\mathrm{R}$ \\
\hline Porfiado (2) & 24 & 30,0 & 56 & 70,0 & 80 \\
Triste (14) & 21 & 26,9 & 57 & 73,1 & 78 \\
Pensamiento fijo (15) & 27 & 31,5 & 50 & 64,9 & 77 \\
Ganas de llorar (16) & 22 & 27,5 & 58 & 72,5 & 80 \\
\hline
\end{tabular}

Fuente: Idem Tabla $1 \quad\left(^{*}\right)$ Corresponde al № de pregunta de la encuesta

Tabla 5 - Conductas de protección micro social más frecuentes entre los escolares no consumidores de drogas lícitas de sexto año básico de las escuelas municipalizadas de la comuna de Chiguayante

\begin{tabular}{lccccc}
\hline \multirow{2}{*}{ Conductas } & \multicolumn{6}{c}{ No Consumidores de Drogas Lícitas } \\
& Sin Riesgo & \multicolumn{2}{c}{ Riesgo } & Recuento \\
& $\mathrm{R}$ & $\%$ & $\mathrm{R}$ & $\%$ & \\
\hline Amenazar a otros (5) & 187 & 89,5 & 22 & 10,5 & 209 \\
Aprovecharse de otros (9) & 183 & 90,6 & 19 & 9,4 & 202 \\
Daño objetos ajenos (10) & 178 & 85,2 & 31 & 14,8 & 209 \\
Robar (11) & 194 & 92,8 & 15 & 7,2 & 209 \\
Discusión entre padres (19) & 178 & 84,8 & 32 & 15,2 & 210 \\
Ignorar paradero hijo (20) & 179 & 85,2 & 31 & 14,8 & 210 \\
Ausentarse de clases (23) & 188 & 90,0 & 21 & 10,0 & 209 \\
Incumplimiento tareas (24) & 168 & 80,8 & 40 & 19,2 & 208 \\
\hline Fuente: Idem Tabla 1 & ${ }^{*}$ () Corresponde &
\end{tabular}

Fuente: Idem Tabla $1 \quad\left(^{*}\right)$ Corresponde al № de pregunta de la encuesta

Este estudio confirmó la alta significación estadística entre nivel de riesgo, traducido en distintas probabilidades de ocurrencia del consumo de drogas lícitas e ilícitas y el número de escolares consumidores de drogas lícitas, evidenciando una tendencia, a mayor nivel de riesgo mayor número de alumnos consumidores, y a la inversa $(p=0,000)$. Cabe destacar que fue posible calcular el nivel de riesgo sólo al $56,1 \%$ de los escolares, dado al número de respuestas obtenidas, y que no hubo escolares exentos de riesgo. Los alumnos de alto riesgo fueron el $26,6 \%$ y los de mínimo riego el $42,0 \%$.

Otro aspecto evaluado fue el índice de severidad, el que expresa el grado de intensidad en que está afectado el funcionamiento del sujeto, aplicado a cada escala y al total de ellas, el fue más relevante en las escalas de depresión y emocional.

\section{DISCUSIÓN}

La prevalencia de vida del consumo de alcohol y tabaco obtenido en este trabajo fue de $16,3 \%$ y $18,7 \%$ respectivamente. La prevalencia de vida según el tipo de consumo de droga lícita a nivel nacional, aportada por un estudio realizado en diversas comunas del país ${ }^{(7)}$ fue de un $73,2 \%$ para el alcohol y un $59,2 \%$ para el tabaco, eso sí teniendo en consideración, que esta prevalencia fue calculada en escolares de $8^{\circ}$ básico a $4^{\circ}$ medio. Al comparar los resultados obtenidos con otra de las comunas de la provincia, Concepción, ella muestra cifras igualmente superiores a las encontradas en el presente estudio con una prevalencia de vida para el alcohol en escolares del mismo grupo de un $69,1 \%$, y para el tabaco de un $60,4 \%$.

En el intento de aproximarse a la edad de los escolares, el mismo informe ${ }^{(7)}$ presenta un grupo de edad inferior a los 14 años, el cual presenta una prevalencia de vida a nivel nacional para el alcohol de $52,4 \%$ y para el tabaco de $43,9 \%$, que al ser comparados con los resultados obtenidos en esta investigación son más próximos pero, igualmente superiores. Otro estudio del CONACE realizado a nivel Nacional $2001^{(12)}$ presenta una prevalencia anual y mensual para el consumo de alcohol, en escolares menores de 14 años, de un $42,1 \%$ y $17,8 \%$ y para el tabaco en $40,9 \%$ y $25,7 \%$ respectivamente. Al comparar estas cifras con las de este estudio encontramos similitud en la prevalencia anual pues, ella fue de un $50 \%$ para el alcohol $(p=0,000)$, y para el consumo de tabaco fue de un $55 \%(p=0,064)$. Respecto a la prevalencia mensual es también muy similar si es comparada con aquella alternativa, más de una vez al mes, cuyos porcentajes presentados por este estudio fueron de 16,3\% y $25,1 \%$ para el alcohol y tabaco.

La prevalencia de vida muestra una tendencia distinta de la nacional, es decir más alta la del consumo 
de tabaco respecto al alcohol, pero la prevalencia mensual muestra la misma tendencia, es decir, mayor para el consumo de tabaco que para el del alcohol.

La edad entre los 8 y 11 años marcó el inicio del consumo de alcohol y de tabaco, en el $80 \%$ de los que han consumido alcohol y en el $60 \%$ de los que han consumido tabaco. En ambos caso no existe asociación estadística.

La marihuana fue la droga ilícita más consumida por los nueve escolares, tendencia confirmada por otros estudios chilenos liderados por el CONACE ${ }^{(13)}$.

Entre los resultados que destacaron por lo inesperado, estuvo el último promedio de notas, ya que no hubo alumnos consumidores que presentaran entre sus promedios calificaciones insuficientes, más bien eran buenas y muy buenas, hecho que contrasta con antecedentes aportados por los llamados factores de riesgo, que asocian el consumo de drogas al bajo o escaso rendimiento escolar.

La disponibilidad de droga $(p=0,005)$ muestra que un $83 \%$ de escolares se mantiene firme en la postura de no consumirlas, sólo un 4,4\% lo haría si dispusiera de ellas, y de éste un 1,4\% corresponde a alumnos que aún no han consumido. De éstos posibles iniciadores en el consumo de drogas, el 84,6\% correspondería a varones $(0,005)$ y las edades de los interesados estaría preferentemente entre los 12 y 11 años, con un 54\% y $16 \%$ respectivamente $(p=0,000)$.

El consumo por otro familiar corroboró que ambas formas de consumo, es decir, alcohol o tabaco independientes, o alcohol más tabaco, estuvieron precedidas por un modelo. ( $p=0,27)$ Pero, un vasto grupo de escolares no consumidores de drogas lícitas sí estuvo expuesto a un modelo, aunque desconocido para quién realizó este estudio. El padre y los hermanos influyen fuertemente a través del refuerzo y modelaje ${ }^{(5)}$.

Las conductas calificadas de riesgo en los escolares consumidores de este estudio, estuvieron preferentemente centralizadas en la escala de depresión y emocional, mientras que la escala académica, familia y conducta centralizó preferentemente las conductas de protección en el grupo de no consumidores. Entre los factores más importantes para desencadenar el uso de drogas en adolescentes, en escuelas públicas de Brasil, han sido las emociones y los sentimientos asociados a intenso sufrimiento psíquico, como depresión, culpa, ansiedad y baja autoestima ${ }^{(14)}$. Por otra parte, un estudio sobre síntomas asociados al consumo de drogas en adolescentes costarricenses mostró que los estudiantes que reportan consumo de alcohol y de tabaco tenían el doble de probabilidad de presentar síntomas de depresión ${ }^{(15)}$. Las conductas como frecuentes ganas de llorar y sentirse triste, estuvieron también a la base de sus resultados. Por lo tanto, la información obtenida en este estudio, sólo viene a confirmar aquellas conductas que pueden ser predictivas del consumo de drogas y por lo tanto base para programas locales de prevención.

Se les pudo calcular los niveles de riego sólo al $57 \%$ de los alumnos, todos ellos presentaron algún grado de riesgo. La tendencia es que a mayor nivel de riesgo mayor número de alumnos consumidores $(p=0,000)$.

Los índices de severidad mayores estuvieron es las escalas de depresión y emocional, (sig $=0,001$ y 0,000 respectivamente) lo que es coincidente con los datos obtenidos. Las conductas de mayor riesgo fueron aquellas que pertenecían a estas escalas.

Entre las limitaciones del estudio estuvo el realizar una encuesta autoaplicada, quizás una encuesta personal habría asegurado una mayor comprensión de las preguntas evitando así inhabilitación de respuestas, pese a que en su momento se realizó la prueba piloto sin que ella evidenciara falta de comprensión por parte de los alumnos. Se percibe una segunda limitación y ella se refiere a que hubo escolares y familias de escolares que se negaron a responder la encuesta, evidenciando un sesgo, pues era posible que esos escolares o sus respectivos familiares fuesen precisamente consumidores. Por lo tanto hubo variables que no fueron consideradas y otras que faltó precisar.

Es importante realizar investigaciones destinadas a elaborar instrumentos que permitan la detección cada vez más precoz de circunstancias favorables al consumo, además de aquellas que evalúen el impacto de las diversas formas de prevención y tratamiento.

\section{CONCLUSIONES}

Conclusión, según los objetivos propuestos, se confirma la existencia de una población escolar consumidora de drogas lícitas con edad de 11 años en el $45 \%$ de los casos. La edad de inicio en el consumo de drogas lícitas se presentó sobre el $60 \%$ de los casos entre los 8 y 11 años. Los resultados respecto del consumo de 
drogas ilícitas no han parecido confiables por las razones antes expuestas. Respecto a las variables estudiadas que dicen relación a los factores de riesgo y de protección, ellas sólo vienen a confirmar lo que tantos notables dicen respecto del tema. Conductas personales a nivel de las emociones estuvieron relacionadas a factores de riesgo, y conductas micro sociales relacionadas con la familia y la escuela fueron parte de los factores de prevención de este grupo escolar. El cálculo de los niveles de riesgo acusó una población escolar sometida a diversos grados de riesgo de consumir drogas.

Por tanto, es necesario comprometer a los más diversos actores de la sociedad en la creación e innovación de estrategias de prevención primaria, secundaria y terciaria, que permita fortalecer a aquellos jóvenes no consumidores y detectar precozmente los ya consumidores, para evitar la consolidación de la adicción y detener la progresión del daño y de las secuelas.

\section{AGRADECIMIENTOS}

Se agradece a la Organización de los Estados Americanos (OEA) y a la Comisión Interamericana para el Control del Abuso de Drogas (CICAD) en la persona de la Coordinadora del Proyecto de las Escuelas de Enfermería en la Reducción de la Demanda de Drogas en América Latina, señora Maria da Gloria Miotto Wright. Los

\section{REFERENCIAS BIBLIOGRÁFICAS}

1. Morrison M. Fundamentos de enfermería en salud mental. Madrid: Hartcourt Brace/Mosby; 1999.

2. Florenzano R, Sotomayor P, Otava M. Estudio comparativo del rol de la socialización familiar y factores de personalidad en las farmacodependencias juveniles. Rev Chil Pediatr 2001; 72(3):219-23.

3. Vallejo J. Introducción a la psicopatología y la psiquiatría. 4a ed. Barcelona: Masso; 1998.

4. Madrigal E, Sayago B. Manual habilidades para la vida: prevención para el consumo de tabaco, alcohol y otras drogas. Caracas: Exlibris; 1999.

5. McWhirter P, Florenzano R, Florenzano M. Correlatos psicosociales de las farmacodependencias: resultados de un estudio en Santiago de Chile. Rev Psiquiatría 1998; 15(1):10-22.

6. Montenegro H. La psiquiatría del niño y del adolescente. Rev Chil Neuropsiquiatr 2002; 52:143-6.

7. CONACE. Estudio del Consumo de Drogas en Población Escolar de Chile, a Nivel Comunal, 1999. Informe Final. Patrocinado por el Ministerio de Educación, Ministerio de Salud y ejecutado por la Escuela de Salud Pública de la Facultad de Medicina de la Universidad de Chile. Chile; 2000. agradecimientos son extensivos a Escuela de Enfermería de Ribeirão Preto, de la Universidade de São Paulo, Brasil, Centro Colaborador de la OMS para el desarrollo de la investigación en enfermería. También se agradece a la profesora guía de esta investigación, señora Ana María Pimenta Carvalho, Doctora en Psicologia, profesora del Departamento de Enfermería Psiquiátrica y Ciências Humanas, de la Escuela de Enfermería de Ribeirão Preto, de la Universidad de São Paulo, y los profesores de la Universidad de Concepción que han contribuido a la realización de la investigación con sus específicos aportes: señor Urcesino González Rubilar, Magíster en Estadística Matemática, Jasna Stipovich Bertoni, Doctora en Enfermería, y Mónica Burgos Moreno, Magíster en Enfermería Médico Quirúrgico.

Agradecemos a todos los docentes de la Escuela de Enfermería de Ribeirão Preto y a los treinta y tres participantes de nueve países latinoamericanos que participaron en el proceso de implementación de los programas, y también al Gobierno del Japón y al Programa de Becas de la OEA por su apoyo financiero y becas que posibilitaron la implementación del "I Programa Regional de Capacitación en Investigación para Enfermeros de América Latina".

8. CONACE. IV Estudio nacional del consumo de drogas en Chile. 2000. Investigación a cargo del centro de Estudios Sociológicos de la Pontificia Universidad Católica de Chile DESUC. Chile; 2000.

9. Macia A. Las drogas: conocer y educar para prevenir. $3^{\circ}$ ed. Madrid: Pirámide. Colección ojos solares; 1997.

10. Florenzano R, Carvajal C, Weil P. Psiquiatría. Santiago (Chile): Mediterráneo; 1999

11. Murrelle L, Sandí L, Díaz A. Cuestionario sobre hábitos, actividades y relaciones de los jóvenes. Rev Ciencias Sociales 1977; 77:87-95.

12. CONACE. Estudio nacional de consumo de drogas en la población escolar 2001 de 8 básico a 4 medio. 2001 . Investigación a cargo del Centro Internacional de Coordinación y Ejecución de Estudios Colaborativos CICEEC y Universidad de Chile. Chile; 2001.

13. CONACE. Consumo de drogas ilícitas en población escolar de Chile. 1999. Ministerio de Educación y Ministerio de Salud. Chile; 1999.

14. Roselli A, Cruz M. O adolescente e o uso de drogas. Rev Bras Psiquiatr 2000; 22(Supl II):32-6.

15. Sandí L, Díaz A. Síntomas de rebeldía, depresión y aislamiento asociados al consumo de drogas en adolescentes costarricenses. Acta Pediatr Costarric 1995; 9:99-102. 